\title{
Front Matter: Volume 10188
}

, "Front Matter: Volume 10188," Proc. SPIE 10188, Radar Sensor Technology XXI, 1018801 (25 May 2017); doi: 10.1117/12.2281094

SPIE. Event: SPIE Defense + Security, 2017, Anaheim, CA, United States 


\title{
PROCEEDINGS OF SPIE
}

\section{Radar Sensor Technology XXI}

\author{
Kenneth I. Ranney \\ Armin Doerry \\ Editors
}

10-12 April 2017

Anaheim, California, United States

Sponsored and Published by

SPIE 
The papers in this volume were part of the technical conference cited on the cover and title page. Papers were selected and subject to review by the editors and conference program committee. Some conference presentations may not be available for publication. Additional papers and presentation recordings may be available online in the SPIE Digital Library at SPIEDigitalLibrary.org.

The papers reflect the work and thoughts of the authors and are published herein as submitted. The publisher is not responsible for the validity of the information or for any outcomes resulting from reliance thereon.

Please use the following format to cite material from these proceedings:

Author(s), "Title of Paper," in Radar Sensor Technology XXI, edited by Kenneth I. Ranney, Armin Doerry, Proceedings of SPIE Vol. 10188 (SPIE, Bellingham, WA, 2017) Seven-digit Article CID Number.

ISSN: 0277-786X

ISSN: 1996-756X (electronic)

ISBN: 9781510608771

ISBN: 9781510608788 (electronic)

Published by

SPIE

P.O. Box 10, Bellingham, Washington 98227-0010 USA

Telephone +1 3606763290 (Pacific Time) · Fax +1 3606471445

SPIE.org

Copyright @ 2017 , Society of Photo-Optical Instrumentation Engineers.

Copying of material in this book for internal or personal use, or for the internal or personal use of specific clients, beyond the fair use provisions granted by the U.S. Copyright Law is authorized by SPIE subject to payment of copying fees. The Transactional Reporting Service base fee for this volume is $\$ 18.00$ per article (or portion thereof), which should be paid directly to the Copyright Clearance Center (CCC), 222 Rosewood Drive, Danvers, MA 01923. Payment may also be made electronically through CCC Online at copyright.com. Other copying for republication, resale, advertising or promotion, or any form of systematic or multiple reproduction of any material in this book is prohibited except with permission in writing from the publisher. The CCC fee code is 0277-786X/17/\$18.00.

Printed in the United States of America.

Publication of record for individual papers is online in the SPIE Digital Library.

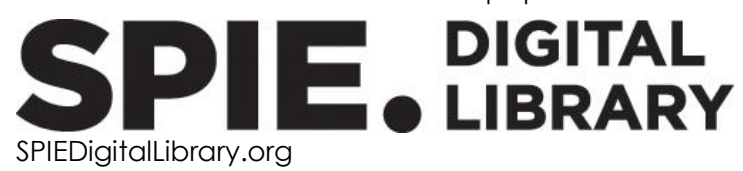

Paper Numbering: Proceedings of SPIE follow an e-First publication model. A unique citation identifier (CID) number is assigned to each article at the time of publication. Utilization of CIDs allows articles to be fully citable as soon as they are published online, and connects the same identifier to all online and print versions of the publication. SPIE uses a seven-digit CID article numbering system structured as follows:

- The first five digits correspond to the SPIE volume number.

- The last two digits indicate publication order within the volume using a Base 36 numbering system employing both numerals and letters. These two-number sets start with 00, 01, 02, 03, 04, 05, 06, 07, 08, 09, OA, OB ... OZ, followed by 10-1Z, 20-2Z, etc. The CID Number appears on each page of the manuscript. 


\title{
Contents
}

\author{
vii Authors \\ ix Conference Committee
}

\section{APPLICATIONS AND EXPLOITATION I}

1018802 Passive coherent location direct signal suppression using hardware mixing techniques [10188-1]

1018803 Multistatic passive coherent location resource optimization [10188-2]

1018804 Continuous high PRF waveforms for challenging environments [10188-3]

1018805 Automatic change detection using very high-resolution SAR images and prior knowledge about the scene [10188-4]

1018806 On results using automated wideband instrumentation for radar measurements and characterization [10188-5]

1018807 Mapping detailed 3D information onto high resolution SAR signatures [10188-6]

INDOOR AND URBAN IMAGING

1018808 A low-cost through-wall FMCW radar for stand-off operation and activity detection [10188-7]

1018809 A coherent through-the-wall MIMO phased array imaging radar based on time-duplexed switching [10188-8]

$10188 \mathrm{OA}$ Theoretical considerations for a dynamic calibration target for through-wall and throughrubble motion-sensing Doppler radar [10188-9]

$10188 \mathrm{OB}$ Detection and tracking of human targets in indoor and urban environments using throughthe-wall radar sensors [10188-10]

\section{QUANTUM RADAR}

10188 OC Electric and magnetic target polarization in quantum radar [10188-11]

10188 OD The Lemur Conjecture [10188-12]

10188 OE Quantum geodesy [10188-13] 

10188 OF Quantum synthetic aperture radar [10188-14]
$101880 G$ Quantum imaging for underwater arctic navigation [10188-15]
$10188 \mathrm{OH}$ Passive ghost imaging using caustics modeling [10188-16]
10188 ol Enhanced sensing and communication via quantum networks [10188-17]

\section{APPLICATIONS AND EXPLOITATION II}

10188 0J Signal processing techniques for the U.S. Army Research Laboratory stepped frequency ultra-wideband radar [10188-18]

10188 OK RFID antenna design for circular polarization in UHF band [10188-20]

$10188 \mathrm{OL}$ VideoSAR collections to image underground chemical explosion surface phenomena [10188-21]

$10188 \mathrm{OM}$ Noise and LPI radar as part of counter-drone mitigation system measures [10188-22]

10188 ON Determining the coherence matrix for single look polarimetric SAR data [10188-23]

\section{COMPONENTS AND TECHNOLOGY}

1018800 Does the central limit theorem always apply to phase noise? Some implications for radar problems [10188-24]

10188 OP Reconfigurable signal processor designs for advanced digital array radar systems [10188-25]

$10188 \mathrm{OQ}$ Linear chirp phase perturbing approach for finding binary phased codes [10188-26]

\section{MICRODOPPLER}

10188 OR The operator approach to the non-uniform Doppler to radar: implications for signal processing [10188-27]

10188 OS Advanced Doppler radar physiological sensing technique for drone detection [10188-28]

10188 OT Millimeter-wave micro-Doppler measurements of small UAVs [10188-29]

10188 OU Micro-Doppler extraction of a small UAV in a non-line-of-sight urban scenario [10188-30]

$10188 \mathrm{OV}$ Classification of micro-Doppler signatures of human aquatic activity through simulation and measurement using transferred learning [10188-31] 
10188 OW Fly Eye radar: detection through high scattered media [10188-32]

10188 0X A solid state 94-GHz FMCW Doppler radar demonstrator for cloud profiling [10188-33]

10188 OY loSiS: a radar system for imaging of satellites in space [10188-34]

$10188 \mathrm{OZ}$ A prototype fully polarimetric 160-GHz bistatic ISAR compact radar range [10188-35]

PROGRAMS AND SYSTEMS II

1018810 Handheld microwave bomb-detecting imaging system [10188-37]

1018812 System upgrades and performance evaluation of the spectrally agile frequencyincrementing reconfigurable (SAFIRE) radar system [10188-39]

\section{PROFILES IN RADAR EDUCATION}

1018816 Radar research at The Pennsylvania State University Radar and Communications Laboratory (Invited Paper) [10188-43]

1018817 Radar research at the University of Kansas (Invited Paper) [10188-44]

NONLINEAR AND COGNITIVE RADAR

1018818 Cognitive software defined radar: waveform design for clutter and interference suppression [10188-45]

1018819 Comparison of RF spectrum prediction methods for dynamic spectrum access [10188-46]

$101881 \mathrm{~A} \quad$ Linearizing an intermodulation radar transmitter by filtering switched tones [10188-47]

\section{POSTER SESSION}

10188 1C An architecture for pre-warping general parametric frequency-modulated radar waveforms [10188-49]

10188 1D Using coherence as a quality measure for complex radar image compression [10188-50]

$101881 \mathrm{E} \quad$ Discriminating spurious signals in radar data using multiple channels [10188-51]

10188 IF Use of unmanned SAR and EO/IR sensor suites for monitoring wildfires [10188-52]

$101881 G$ Classification of radar jammer FM signals using a neural network [10188-53] 
$10188 \mathrm{1H}$ Optimization of neural network architecture for classification of radar jamming FM signals [10188-54]

1018811 Using TerraSAR-X satellite data to detect road age and degradation [10188-55]

$10188 \mathrm{IJ} \mathrm{Initial} \mathrm{processing} \mathrm{and} \mathrm{analysis} \mathrm{of} \mathrm{forward-and} \mathrm{side-looking} \mathrm{data} \mathrm{from} \mathrm{the} \mathrm{spectrally} \mathrm{agile}$ frequency-incrementing reconfigurable (SAFIRE) radar [10188-56]

$101881 \mathrm{~K}$ A quantum inspired model of radar range and range-rate measurements with applications to weak value measurements [10188-57]

$10188 \mathrm{1L}$ Design and analysis of a multi-passband complex filter for the multiband cognitive radar system [10188-58]

10188 1M Radar detection of buried targets in coastal environments [10188-59]

$10188 \mathrm{iN}$ Low-elevation tracking technique for X-band unmanned aerial vehicle automatic take-off and landing system [10188-60]

1018810 Bearing angle estimation based on synthetic aperture radar (SAR) image [10188-61] 


\section{Authors}

Numbers in the index correspond to the last two digits of the seven-digit citation identifier (CID) article numbering system used in Proceedings of SPIE. The first five digits reflect the volume number. Base 36 numbering is employed for the last two digits and indicates the order of articles within the volume. Numbers start with 00, 01, 02, 03, 04, 05, 06, 07, 08, 09, OA, OB...0Z, followed by 10-1Z, 20-2Z, etc.

Addison, Stephen R., 00

Alkhazraji, Emad, OK

Allen, Christopher, 17

Andersson, Åsa, OU

Anger, S., OY

Anglberger, H., 05, 07

Arnold, Emily, 17

Beaudoin, C. J., $0 Z$

Bickel, D. L., 1D, $1 \mathrm{E}$

Blasch, Erik, 10

Blunt, Shannon D., 17, 18

Boutte, David, OB

Brandsema, Matthew J., OA, OC

Brennan, Paul, 09

Brode, Chad M., 1M

Calloway, Terry M., OL

Chen, Genshe, 10

Chen, Qingchao, 08, 09

Chetty, Kevin, 08, 09

Cho, M.-H., IN

Christianson, Andrew J., 02, 03

Clark, John T., 12, $1 \mathrm{~J}$

Corbeil, Allan, 04

Coulombe, M. J., OZ

DeMartinis, G., $0 Z$

Dill, S., OY

Doerry, A. W., 1C, 1D, 1E, 1F

Dogaru, Traian, 06

Dunkel, R., $1 \mathrm{~F}$

Escalante, George, 1K

Flores, Benjamin C., 1G, 1H

Galanos, Daniel T., 12

Gallagher, Kyle A., 19

Garcia Carrillo, Luis R., OS

Gatesman, A. J., OZ

Geaga, Jorge V., ON

Gonwara, Ashok, 0W, 10

Govoni, Mark A., 06

Goyette, T., $0 Z$

Gray, John E., 0O, OR

Gustavsson, Magnus, OU

Hale, Richard, 17

Hansen, Jeremiah J., OR

Harner, Michael J., OA

Harrison, Arthur C., 12, $1 \mathrm{~J}$

Horgan, T., $\mathrm{OZ}$

Hu, W.-Y., $1 \mathrm{~N}$

Huang, Yih-Ru, OM

Hui, Rongqing, 17
Hunter, Robert I., OX

Irshad, Usama Bin, OK

Jaroszewski, Steven, 04

Javaid, Muhammad Sharjeel, OK

Jendzurski, John R., OA

Jirousek, M., OY

Jitrik, Oliverio, $\mathrm{OD}, \mathrm{OE}, \mathrm{OF}, \mathrm{OH}$

Johansson, Tommy, OU

Jonsson, Rolf, OU

Kaiser, Sean A., 02, 03

Kanugo, Jayanth, $\mathrm{OH}$

Karlsson, Nils, OU

Kempf, T., 05

Keshmiri, Shahriar, 17

Khan, Muhammad Talal Ali, OK

Kim, Youngwook, OV

Kirk, Benjamin H., 18

Kirose, Getachew A., 12, $1 \mathrm{~J}$

Kovarskiy, Jacob A., 19

Lanzagorta, Marco, OC, OD, OE, OF, OG, $\mathrm{OH}$

Le, Calvin, 06

Lee, Hua, OB

Lee, Hua-Chin, $1 \mathrm{~L}$

Leuschen, Carlton, 17

$\mathrm{Li}$, Bing C., OQ

Li, Jilu, 17

Lin, M.-Y., IN

Lin, S.-Y., IN

Lok, Lai Bun, 09

Longepe, Nicolas, 11

Martone, Anthony F., 18, 19, 1A

Mazzaro, Gregory J., 1A

Mendoza, Ariadna, 1G, 1H

Molchanov, Pavlo, OW, 10

Moon, Taesup, OV

Narayanan, Ram M., 02, 03, 0A, 0C, 12, 16, 18, $19,1 \mathrm{~J}, 1 \mathrm{M}$

Necsoiu, Marius, 11

Nguyen, Lam, 0J, $1 \mathrm{~J}$

Nilsson, Stefan, OU

Nixon, William E., $0 Z$

Owen, Jonathan W., 18

Paden, John, 17

Parashar, Aditya, $\mathrm{OH}$

Park, Jinhee, OV

Parks, Allen D., OR

Parra, Jorge O., 11

Paulter, Nicholas G., OA

Peichl, M., OY 
Pham, Khanh, 10

Phelan, Brian R., 12, $1 \mathrm{~J}$

Radzicki, Vincent R., OB

Rahman, Samiur, OT

Ranney, Kenneth I., 12, 1A, $1 \mathrm{~J}$

Ressler, Marc A., 12, $1 \mathrm{~J}$

Ritchie, Matthew, 08, 09

Robertson, Duncan A., OT, OX

Rodriguez-Morales, Fernando, 17

Ryland, Robert, 04

Saddler, R., IF

Salandrino, Alessandro, 17

Saponaro, Philip J., Jr., 12

Schreiber, E., OY

Shahid, Hamza, OK

Sherbondy, Andrew J., 1 A

Sherbondy, Kelly D., 12, 18, 19, 1A, 1J

Smith, Gregory, $1 \mathrm{~J}$

Smith, James F., III, OI

Sobczak, Kevin, 06

Sobota, David, 04

Soto, Alberto, 1G, 1H

Speck, R., 05, 07

Stiles, James, 17

Stilla, U., 05

Suarez, Hernan, OP

Sun, J.-S., $1 \mathrm{~N}$

Taylor, Paul, OB

Tayyab, Umais, OK

Thumann, Charles, $0 \mathrm{M}$

Ting, Der-Hong, $1 \mathrm{~L}$

Treible, Wayne R., 12

Tsao, Ya-Lan, $1 \mathrm{~L}$

Uhlmann, Jeffrey, OD, OE, OF, OH

Venegas-Andraca, Salvador E., OD, OE, OF, OH

Villamil Lopez, C., 05

Wahl, Daniel E., OL

Walter, Gary R., 11

Wang, Zhonghai, 10

Woodbridge, Karl, 08, 09

Xiang, Xingyu, 10

$\mathrm{Xu}, \mathrm{HaO}, \mathrm{OS}$

Yocky, David A., OL

Yoon, Ji Hwan, OS

Yu, Xining, OP

Zhang, Yan (Rockee), OM, OP

Zhao, Ming, $\mathrm{OH}$ 


\title{
Conference Committee
}

\author{
Symposium Chair
}

Donald A. Reago Jr., U.S. Army Night Vision \& Electronic Sensors

Directorate (United States)

Symposium Co-chair

Arthur A. Morrish, Raytheon Space and Airborne Systems

(United States)

Conference Chairs

Kenneth I. Ranney, U.S. Army Research Laboratory (United States)

Armin Doerry, Sandia National Laboratories (United States)

Conference Program Committee

Fauzia Ahmad, Temple University (United States)

Moeness G. Amin, Villanova University (United States)

Joseph C. Deroba, U.S. Army CERDEC Intelligence and Information

Warfare Directorate (United States)

Mark Govoni, U.S. Army CERDEC Intelligence and Information

Warfare Directorate (United States)

John E. Gray, Naval Surface Warfare Center Dahlgren Division

(United States)

Majeed Hayat, The University of New Mexico (United States)

Volker Horndt, General Atomics Aeronautical Systems, Inc.

(United States)

Chandra Kambhamettu, University of Delaware (United States)

Seong-Hwoon Kim, Raytheon Space \& Airborne Systems (United States)

Marco O. Lanzagorta, U.S. Naval Research Laboratory (United States)

Changzhi Li, Texas Tech University (United States)

Jenshan Lin, University of Florida (United States)

Ronald D. Lipps, U.S. Naval Research Laboratory (United States)

David G. Long, Brigham Young University (United States)

Neeraj Magotra, Western New England University (United States)

Anthony F. Martone, U.S. Army Research Laboratory (United States)

Gregory J. Mazzaro, The Citadel-The Military College of South

Carolina (United States)

George J. Moussally, Mirage Systems (United States)

Ram M. Narayanan, The Pennsylvania State University (United States)

Lam H. Nguyen, U.S. Army Research Laboratory (United States) 
Hector A. Ochoa, The University of Texas at Tyler (United States) Thomas Pizzillo, U.S. Naval Research Laboratory (United States)

Zhijun G. Qiao, The University of Texas-Pan American (United States)

Ann M. Raynal, Sandia National Laboratories (United States)

Jerry Silvious, U.S. Army Research Laboratory (United States)

David Tahmoush, U.S. Naval Research Laboratory (United States)

Russell Vela, Air Force Research Laboratory (United States)

Frank Yakos, SELEX Galileo, Inc. (United States)

Yan Zhang, The University of Oklahoma (United States)

Ruolin Zhou, Western New England University (United States)

\section{Session Chairs}

1 Applications and Exploitation I

Seong-Hwoon Kim, Raytheon Space and Airborne Systems

(United States)

2 Indoor and Urban Imaging

Kyle A. Gallagher, The Pennsylvania State University (United States)

Gregory J. Mazzaro, The Citadel-The Military College of South

Carolina (United States)

3 Quantum Radar

Marco O. Lanzagorta, U.S. Naval Research Laboratory (United States)

Salvador Elias Venegas-Andraca, Tecnológico de Monterrey

(Mexico)

4 Applications and Exploitation II

Ann Marie Raynal, Sandia National Laboratories (United States)

5 Components and Technology

Gregory J. Mazzaro, The Citadel-The Military College of South Carolina (United States)

Brian R. Phelan, The Pennsylvania State University (United States)

6 MicroDoppler

Lam H. Nguyen, U.S. Army Research Laboratory (United States)

7 Programs and Systems I

Mark A. Govoni, U.S. Army CERDEC Intelligence and Information Warfare Directorate (United States)

David Tahmoush, U.S. Naval Research Laboratory (United States) 
8 Programs and Systems II

Mark A. Govoni, U.S. Army CERDEC Intelligence and Information Warfare Directorate (United States)

David Tahmoush, U.S. Naval Research Laboratory (United States)

9 Keynote Session

Kenneth I. Ranney, U.S. Army Research Laboratory (United States) Armin W. Doerry, Sandia National Laboratories (United States)

10 Profiles in Radar Education

Kenneth I. Ranney, U.S. Army Research Laboratory (United States)

Armin W. Doerry, Sandia National Laboratories (United States)

11 Nonlinear and Cognitive Radar

Ram M. Narayanan, The Pennsylvania State University (United States) 
Proc. of SPIE Vol. 10188 1018801-12 Downloaded From: https://www.spiedigitallibrary.org/conference-proceedings-of-spie on 26 Apr 2023
Terms of Use: https://www.spiedigitallibrary.org/terms-of-use 